\title{
Écosystème
}

\section{L'intelligence artificielle ou le bouleversement de la représentation}

\section{Maud Maffei}

Volume 3, numéro 1, 2021

Intelligence artificielle

URI : https://id.erudit.org/iderudit/1075896ar

DOI : https://doi.org/10.7202/1075896ar

Aller au sommaire du numéro

\section{Éditeur(s)}

La chambre blanche

ISSN

2562-3222 (numérique)

Découvrir la revue

Citer cet article

Maffei, M. (2021). L'intelligence artificielle ou le bouleversement de la représentation. Écosystème, 3(1), 69-80. https://doi.org/10.7202/1075896ar

\section{Résumé de l'article}

Aujourd'hui, les artistes interrogent à différents niveaux les enjeux de l'intelligence artificielle sur nos vies, poursuivant un thème en fait millénaire : l'effroi et la fascination face aux capacités des machines que produit l'humain. Dans la continuation de l'art génératif des années 1960, les oeuvres utilisant l'intelligence artificielle aujourd'hui s'interrogent sur les enjeux actuels de ses sophistications algorithmiques : notamment la place du hasard que cette technologie implique et les espaces-temps infinis qu'elle produit, nous donnant à voir une multiplicité de mondes possibles. À travers l'analyse des oeuvres des artistes Hito Steyerl, Gregory Chatonsky et Trevor Paglen, Maud Maffei voit l'intelligence artificielle comme une sorte de cristal où les réels se démultiplient, s'imbriquent, se réverbèrent les uns sur les autres. Les réalités alternatives que ces artistes nous dévoilent dans notre présent peuvent nous aider à le comprendre, le penser, l'anticiper. Elles nous ramènent aussi aux forces les plus essentielles de l'intelligence humaine face à la machine : l'esprit critique et la capacité à changer les règles de tout système. 


\section{Maud Maffei}

\section{L'intelligence artificielle ou le bouleversement de la représentation}

Résumé

Aujourd'hui, les artistes interrogent à différents niveaux les enjeux de l'intelligence artificielle sur nos vies, poursuivant un thème en fait millénaire : l'effroi et la fascination face aux capacités des machines que produit l'humain.

Dans la continuation de l'art génératif des années 1960, les œuvres utilisant l'intelligence artificielle aujourd'hui s'interrogent sur les enjeux actuels de ses sophistications algorithmiques : notamment la place du hasard que cette technologie implique et les espaces-temps infinis qu'elle produit, nous donnant à voir une multiplicité de mondes possibles. À travers l'analyse des œuvres des artistes Hito Steyerl, Gregory Chatonsky et Trevor Paglen, Maud Maffei voit l'intelligence artificielle comme une sorte de cristal où les réels se démultiplient, s'imbriquent, se réverbèrent les uns sur les autres. Les réalités alternatives que ces artistes nous dévoilent dans notre présent peuvent nous aider à le comprendre, le penser, l'anticiper. Elles nous ramènent aussi aux forces les plus essentielles de l'intelligence humaine face à la machine : l'esprit critique et la capacité à changer les règles de tout système. 
«Alors que le futur était prédit, le présent devint imprédictible. ${ }^{1}$

« II devient plus intéressant de voir ce que fait un clown sur une corde tendue plutôt que ce que fait un simple funambule. Vous pouvez toujours prévoir ce que le funambule fera, c'est un processus mécanique. Mais vous ne pourrez jamais prévoir ce que le clown va faire. ${ }^{2}$ Robert Smithson

Les scénarios de films où l'être humain ne se distingue plus de la machine et où il se fait avoir par elle se retrouvent à profusion depuis la révolution électronique, de 2001 l'Odyssée de l'espace (1968), à The Matrix (1999) en passant par Blade Runner (1982). Ils traitent à la fois de la quête d'éternité de l'être humain et de ses peurs de devenir les machines qu'il produit avec ardeur pour tendre vers cette éternité.

Notamment, dans Creation of the humanoïds, un film de 1964 - dont l'artiste Robert Smithson fait remarquer qu'il s'agit du film préféré d'Andy Warhol $^{3}$ - l'âme d'un être humain peut durant un certain délai après sa mort être transférée dans un robot en tous points semblable en apparence àl'humain si bien que lui-même ne peut savoir qu'il n'est plus humain. Invulnérable, le robot doit néanmoins être remplacé tous les 150 ans, l'âme pourra poursuivre une « vie » éternelle en étant alors transférée dans un nouveau robot.

Ce que l'on appelle aujourd'hui " intelligence artificielle " se développe au moment où émergent de tels films de science-fiction, elle en est la source d'inspiration.

Les progrès actuels de l'intelligence artificielle nous font projeter de grands espoirs : pourra-t-elle nous sauver de la catastrophe climatique? Nous aider à trouver des solutions pour garantir de l'eau et une alimentation durable pour toute la planète? Prévenir les maladies et traiter les handicaps? Ou encore, trouver l'âme sœur et l'amour fou?

\footnotetext{
${ }^{1}$ Traduction libre de « But as the future was predicted, the present became unpredictable », Hito Steyerl dans This is the Future, 2019, installation vidéo avec images réalisées par intelligence artificielle.

${ }^{2}$ Traduction libre de " It becomes more interesting to see what a clown does with tightrope walking rather than the tightrope walker does. You can always expect what a tightrope walker will do - But you can never expect what the clown will do. So I guess it's that unexpected aspect that's always turning up and turning against itself. The limits are always against themselves. As soon as you think you have the limits established they're there; but then again they're not there. " dans " Interview with Patricia Norvell », Recording Conceptual Art, ed. par Alexander Alberro et Patricia Norvell, Berkeley, University of California Press, 2001, pp. 132-133.
}

${ }^{3}$ Dans « Entropy and the new monuments (1966) », dans The Collected Writings of Robert Smithson, ed. par Jack Flam, Berkeley, University of California Press, 1996, p. 424 
Depuis ses débuts, l'intelligence artificielle (IA) exerce à la fois fascination et effroi : fascination pour toutes les prouesses qu'elle permet, puis effroi face au fait qu'elle pourrait nous détruire lorsqu'elle aura atteint un certain niveau ou lors d'un bogue, souvenons-nous d'Al, le robot de 2001 l'Odyssée de l'espace.

Comment les artistes travaillent-ils aujourd'hui avec l'intelligence artificielle? Que révèlent-ils de nos rapports à cette technologie?

\section{Cadrer le hasard : l'art et les développements de l'intelligence artificielle}

Dès les années 1960, les artistes pensent des œuvres qui résonnent avec les enjeux de l'électronique et intègrent l'aléatoire dans leurs pratiques : soit en travaillant directement avec les machines électroniques, soit par résonance. Lorsqu'en 1959 Jean Tinguely réalise ses méta-matics, des machines mécaniques à dessiner, le tracé du dessin confié à la machine est simplement encadré, il n'est pas contrôlé : Tinguely crée la machine qu'il laisse ensuite exécuter des tracés aléatoires en gribouillis. Ce qui compte, c'est la machine en tant que sculpture en action, on sort du dessin comme "dessein ", un tracé intentionnel.

Lorsqu'ils réalisent des dessins assistés par ordinateur, Vera Molnar et Gottfried Honegger (respectivement dès 1968 et 1970) reviennent à un tracé intentionnel du dessin : la machine dessine à partir du programme créé par l'artiste. Avec le programme informatique, l'artiste structure une trame contrôlée dans laquelle la machine exécute les tracés avec une certaine latitude aléatoire.

En 1968, Sol LeWitt met en place ses Wall Drawings par lesquels il introduit en arts visuels ce qui était réservé au domaine de la musique : la partition. Les Wall Drawings sont réalisés par des interprètes à partir de la partition de l'œuvre, que l'on appelle aussi son énoncé, sa notice d'instruction. II s'agit du code de l'œuvre qui peut être réalisée à l'infini avec variations en fonction de l'interprétation des exécutants et du lieu de destination.

Ces exemples mettent en évidence un enjeu artistique clé : le hasard contrôlé. L'artiste travaille depuis toujours avec des outils dont il sonde les limites, avec lesquels il joue, qu'il contrôle : la tavoletta, la camera obscura, l'appareil photo, la caméra... Les algorithmes constituent un nouveau médium artistique depuis 
les années 1960 avec ce que l'on appelle " l'art génératif », soit des œuvres dont les formes sont programmées dans leur structure et se développent en suivant cette structure avec variations à l'infini. Les programmes actuels d'intelligence artificielle ne sont ainsi que des sophistications algorithmiques qui reflètent de nouvelles interrogations. II s'agit alors pour les artistes de travailler avec la part de hasard que ces outils impliquent en la cadrant dans la conceptualisation de leurs œuvres.

\section{Le Generative Adversarial Network : un nouveau dispositif de représentation}

Le type d'intelligence artificielle aujourd'hui le plus répandu est celui de l'apprentissage automatique (Machine Learning) qui fonctionne par des réseaux adverses génératifs (Generative Adversarial Network, GAN). Mis en place en 2014 par le chercheur en informatique lan Goodfellow, il s'agit d'un système complexe qui fait dialoguer deux programmes de réseaux neuronaux : un générateur construit des formes à partir de données qui lui sont transmises tandis qu'un discriminateur revoit les formes créées pour les adapter à d'autres normes. II s'agit d'une rétroaction, soit le principe clé de la cybernétique : l'information est réanalysée à chaque retour sur ellemême dans le circuit et s'adapte en fonction des autres données. C'est un système dialectique. D'autres types d'intelligence artificielle peuvent se combiner avec le GAN, notamment l'imagerie par résonance magnétique fonctionnelle (Functional Magnetic Resonance Imaging IRMF) très utilisée dans la recherche en sciences cognitives.

La programmation d'un GAN se réalise par la transmission d'un nombre vertigineux de données : il est "éduqué » par l'être humain qui l'utilise et reproduit tous les préjugés humains transmis. On se souvient du chatbot Tay mis au point par Microsoft et qui dût être retiré de la circulation 24h après sa mise en ligne en 2016, car il s'était révélé raciste et homophobe.

Que fait un GAN lorsqu'on lui transmet une multitude d'images? II en réalise une sorte de synthèse en continu, une image en perpétuelle transformation qui constitue une "compression » de toutes les images qui lui ont été transmises. II s'agit alors d'un nouveau type de représentation. Une représentation du réel à la puissance deux, puisqu'elle travaille à partir d'images du réel et non du réel. 
En tant que telle, la technologie du GAN est fascinante et elle est utilisée dans une multitude de domaines. On peut dire que l'IA réalise un type nouveau d' " image cristal ", une sorte de cristal liquide ou cristal en liquéfaction. Un cristal est une matière solide, contractée et dense, c'est souvent le dernier stade géologique d'une matière liquide ou gazeuse, telle la lave de volcan qui passe de l'état liquide à solide en se refroidissant. L'IA reçoit des strates d'images, et en les synthétisant, disons qu'elle les liquéfie. À partir d'images du passé, elle crée une nouvelle image synthétique.

\section{L'image cristal de l'intelligence artificielle}

Par la métaphore cristalline à laquelle fait penser l'IA, il est heureux de découvrir une œuvre qui traite précisément de géologie pour mettre en lumière le bouleversement de la représentation que produit l'IA: Terre Seconde (2019) de l'artiste franco-canadien Grégory Chatonsky.

À la base de son travail, Grégory Chatonsky a recherché des milliers d'images de la planète, images aériennes et géologiques, qu'il a transmises à la machine d'IA. À partir de ces banques d'images de la terre présentant diffrentes échelles et divers types de géologies, l'IA crée les images d'une autre terre, une " terre seconde ". À l'été $2019,{ }^{4}$ au Palais de Tokyo à Paris où le dispositif est installé pour l'exposition $A l t+R$, nous voyons des sortes de falaises se former et s'effondrer. C'est une géologie en mouvement accéléré qui fait écho à l'urgence écologique que nous vivons à notre âge d'anthropocène que Chatonsky préfère appeler, pour être plus direct, " extinction ». Ceci résonne encore avec le cristal, forme ultime d'agencement des atomes d'une matière.

Grégory Chatonsky présente son œuvre comme « un monument à la Terre disparue, à notre civilisation hypermnésique. Tout ce qui resterait serait une machine qui en a toutes les données Internet et qui essaye de se souvenir de tout ce que nous avons été. $»^{5}$

\footnotetext{
${ }^{4}$ Exposition organisée par Gaël Charbau pour les lauréat·e·s du Prix Audi Talents Award : Grégory Chatonsky, Marielle Chabal et Léonard Martin, du 26 juin au 14 juillet 2019.

${ }^{5}$ Grégory Chatonsky dans « L'autre terre de Grégory Chatonsky au Palais de Tokyo » par Laurent Carpentier, dans Le Monde, 27 juin 2019. En ligne, https://www.lemonde.fr/culture/article/2019/06/27/arts-l-autre-terre-de-gregory-chatonsky-au-palais-detokyo_5482045_3246.html, page consultée le 05/02/2021.
} 
À côté de ces images, on trouve des sculptures aux formes organiques : les formes sont également créées par l'IA à laquelle l'artiste transmet des fichiers $3 \mathrm{D}$ d'organismes vivants et de fossiles. Comme les images 2D que nous avons vues, ce sont des synthèses d'organismes passés et présents, " des organismes qui auraient pu exister. C'est une synthèse du passé et en même temps cette synthèse du passé ouvre la voie à une autre possibilité, soit qui n'a pas eu lieu, soit qui pourrait avoir lieu. ${ }^{6}$

Plutôt qu' " intelligence artificielle », Chatonsky parle lui d'" imagination artificielle " dans le sens le plus brut du terme "imagination ", à savoir, la capacité à produire des images, former des images : une imagination mécanique qui selon lui se définit négativement par rapport à l'imagination humaine. Terre seconde est bien une représentation à la puissance deux de la terre dans le sens où il s'agit d'une représentation à partir de représentations (images) de la terre.

L'IA se révèle comme un nouveau dispositif de représentation qui constitue un tournant dans l'histoire de la représentation, à l'image de ce qu'ont été les inventions successives de la photographie et du cinéma au $19^{\text {e }}$ siècle. Un bouleversement du concept de mimésis.

\section{Quel est le temps de l'intelligence artificielle?}

Composée d'images passées, l'intelligence artificielle est-elle une image cristal synthèse du passé, est-elle une image du présent, ou encore une image du futur? C'est la question que pose This is the Future, l'installation vidéo de l'artiste allemande Hito Steyerl présentée à la Biennale de Venise 2019.

Sur les écrans, nous voyons défiler des images réalisées par IA : des fleurs naissent de nulle part et se fondent les unes dans les autres laissant la place à des bribes de paysage vénitien. Les images sont oscillantes, en perpétuelle transformation, rien n'est jamais complètement saisissable.

${ }^{6}$ Dans «Sonder la Terre seconde» de Grégory Chatonsky, par Audi Talents, 6 min., fichier numérique, en ligne, sur https://www.youtube.com/watch?v=JRBkwQwy6n0\&ab_channel=Auditalents, page consultée le 08/02/2021. 
Les narratrices de la vidéo nous guident à travers un jardin en croissance perpétuelle et font ressortir un point caractéristique de l'IA : toutes les images que nous voyons sont des synthèses de données passées qui visent à anticiper le futur, à le prédire. Elles mettent en évidence un fait : notre présent, ici et maintenant nous échappe : " attention, quelles que soient les prédictions du réseau, cela n'arrivera peut-être pas (...) laissez-moi faire une prédiction : rien de tout cela n'arrivera. " ${ }^{7}$ disent-elles. Le jardin artificiel dans lequel nous naviguons est un espace potentiel et incertain en décalage avec un réel que nous ne regardons pas.

Dans son ouvrage Rivers of Shadow : Eadweard Muybridge and the Technological Wild West, Rebecca Solnit montrait comment chaque nouvelle technologie tendait à l' " annihilation du temps et de l'espace ». " [A]nnihiler le temps et l'espace est ce que la plupart des nouvelles technologies aspirent à faire : la technologie considère les termes mêmes de notre existence corporelle comme un fardeau $"{ }^{8}$ déclarait-elle. Le temps et l'espace - en premier lieu le présent - semblent nous glisser des mains. Les artistes traitent de cette question à chaque nouvelle révolution technologique.

« L'absence du présent » est un thème récurrent dans les pratiques des artistes contemporains de la révolution électronique des années 1960-1970, en particulier avec l'utilisation de la rétroaction vidéo. On pense par exemple à Live-Taped Corridor (1970) de Bruce Nauman où en marchant dans le couloir, notre image sur l'écran vidéo au fond nous échappe puisque l'on se voit de dos s'éloignant : l'espace s'étire à l'infini, et ce paradoxalement du fait du retour du signal vidéo sur lui-même. De manière similaire, dans Present Continuous Past (1978) de Dan Graham, les moniteurs en rétroaction avec huit secondes de décalage ne permettent que d'y saisir une image passée de nous-mêmes, on se trouve comme dans un cristal où un espace en réverbère un autre.

Ces œuvres résonnent avec les bouleversements spatio-temporels de la révolution électronique : le retour de l'information sur elle-même dans les réseaux électroniques avec le décalage temporel entre émission et réception de l'information ainsi que la contraction et la dilatation de l'information.

\footnotetext{
${ }^{7}$ Traduction libre de « warning; whatever the network predicts may not be the case (...) let me make a prediction : none of this will ever happen. » dans Hito Steyerl, This is the Future, Biennale de Venise, 2019. Un aperçu de la vidéo ici et la citation est à 12 min. 50 sec. en ligne, https://www.youtube.com/watch?v=AYQB4_riCCg, page consultée le 11/02/2021.

${ }^{8}$ Traduction libre de «annihilating time and space' is what most new technologies aspire to do: technololgy regards the very terms of our bodily existence as burdensome», dans Rebecca Solnit, Rivers of Shadow : Eadweard Muybridge and the Technological Wild West, New York, Penguin Books, 2004.
} 
This is the Future porte en résonance les enjeux de telles œuvres lorsqu'elle met en évidence les bouleversements actuels qu'implique I'IA : quelle est la réalité de notre présent si ce n'est que le lieu où transitent des images synthétiques du passé supposées nous aider à construire un réel à venir?

Par le questionnement du temps auquel appartiennent les images réalisées par IA, This is the Future propose une critique de nos croyances et dépendances en l'IA pour nous renvoyer à nos conditions présentes d'existence. Hito Steyerl présente l'IA comme une pythie du $\mathrm{XXI}^{\mathrm{e}}$ siècle dont il faut savoir garder - c'est le cas face à toute pythie - une certaine distance critique pour pouvoir en décrypter et interpréter les messages.

This is the Future et Terre seconde nous montrent un présent facetté d'autres réels : chaque fois, nous nous retrouvons devant des espaces-temps qui nous présentent une multiplicité de mondes possibles. Le temps des images réalisées par IA est cristallin, il s'agit d'un temps à facettes multiples. Les artistes révèlent l'IA comme un cristal où les réels se démultiplient, s'imbriquent, se réverbèrent entre eux. Ces réalités autres se réfractent dans notre présent, elles peuvent nous aider à le comprendre, le penser, l'anticiper.

\section{L'œil de la machine face à l'œil humain}

Comment voit une machine d'IA? Que voit-elle? Comment est-elle éduquée à voir? Comment utilise-t-elle les données visuelles? II s'agit de questions autour desquelles l'artiste américain Trevor Paglen mène ses recherches depuis plusieurs années.

Avec sa série Adversarially Evolved Hallucination (2017), il déconstruit le processus d'apprentissage du GAN de l'IA pour en montrer les limites en l'amenant dans un " no machine's land " pour ainsi dire. Comme l'humain, I'IA est éduquée à une reconnaissance codée entre mots et choses : une pomme, l'image d'une pomme et le mot " pomme " correspondent. En utilisant I'IA, Trevor Paglen s'attache à perturber ces règles conventionnelles de correspondances entre mots et choses. II voit en ceci dans Magritte un précurseur essentiel. En disant « ceci n'est pas une pipe ${ }^{9}$ à la machine qui a appris à reconnaître des images de pipes, il la fait dérailler et pointe ainsi les différences entre l'humain et la machine.

${ }^{9}$ René Magritte, dans La trahison des images, 1928-1929, peinture à l'huile sur toile, 59 x 65 cm, LACMA, Los Angeles. 
Dans Adversarially Evolved Hallucination, il remplace la correspondance mots-images à laquelle est entraînée la machine par une correspondance par symboles issus de la littérature ou de la philosophie. Paglen relève par exemple les symboles liés à l'interprétation des rêves selon Freud puis les transmet à la machine. Dans un environnement donné, la machine voit alors tout à travers le regard des symboles psychanalytiques : par exemple dans une cuisine plutôt que de voir un café ou un verre, elle verra un fantôme ou un monstre, car Paglen l'aura éduquée à prendre un café pour un fantôme, un verre pour un monstre. Paglen tend ainsi à montrer combien "le degré auquel des métaphores, des subjectivités, des formes très spécifiques de sens commun sont intégrées à ces systèmes. ${ }^{10}$

Une machine d'IA est éduquée pour voir certaines choses dans des objectifs précis, des missiles ou des bombes par exemple, et elle ne saura ni voir autre chose si elle n'y a pas été éduquée, ni se remettre en question, elle n'a pas d'affect. II s'agit d'un nouveau type d'œil — un œil indifférent — dont Paglen met en lumière les décalages avec l'œil humain. À la différence de la machine, l'humain change constamment les règles avec lesquelles il vit : un dessin de feuille devient un canon, dit Magritte dans Les mots et les images, tout comme on aime un jour un objet que l'on n'aime soudain plus le lendemain.

Si l'intelligence humaine est limitée en termes quantitatifs, c'est-à-dire quant au nombre de données qu'elle peut traiter, souvenons-nous qu'elle est illimitée par son versant « disruptif » : elle sait changer les règles. C'est sa singularité, sa force.

\section{Intelligence artificielle, intelligence augmentée, imagination artificielle?}

Terre Seconde, This is the Future et Adversarially Evolved Hallucination interrogent différentes facettes de l'IA pour en révéler les enjeux. Un point fondamental que mettent en évidence les artistes : on retrouve toujours un humain derrière un programme d'intelligence artificielle et la phase d'éducation de la machine, qui consiste à lui transmettre une multitude de données, nécessite beaucoup de travail humain.

Par sa capacité de calcul vertigineuse, l'IA effectue dans tous les domaines de la vie des tâches que nous mettrions un temps incommensurable à réaliser nous-mêmes, mais elle ne saurait remplacer l'intelligence humaine.

\footnotetext{
${ }^{10}$ Traduction libre de « One of the points of those artworks for me is showing the degree to which metaphors and subjectivities and highly specific forms of common sense are built into these systems. " dans «This Is the Project of a More Just World : Trevor Paglen on Making Art That Shows Alternative Realities » de Trevor Paglen, en entrevue avec Brian Boucher, 11 juin 2018 sur artnet, en ligne, https://news.artnet.com/art-world/trevor-paglen-interview-1299836, page consultée le 08/02/2021.
} 
Nous restons ainsi réellement loin des scénarios de films de science-fiction où la machine prend le dessus sur l'être humain : l'intelligence artificielle consciente n'existe pas, elle reste actuellement systématique et ne sait pas user d'analogies autres que celles qui lui ont été transmises. Elle n'est pas indépendante et travaille de pair avec l'esprit humain dans un but humain. Ainsi, au terme d' " intelligence artificielle », certains préfèrent celui d' « intelligence augmentée ». L' « imagination artificielle » au sens le plus brut d'une production artificielle d'images que formule Grégory Chatonsky rejoint cette recherche d'un autre terme.

Reste une question : si les machines en intelligence artificielle se répandent aujourd'hui pour réaliser un certain nombre de tâches de la vie courante, quelles sont leurs influences sur la malléabilité de l'esprit humain? La machine tendrait-elle à le rigidifier, elle qui ne sait pas remettre en question les lois qu'elle suit?

En vivant avec ces machines, l'humain devra peut-être se battre pour ne pas devenir les machines qu'il produit et garder la flexibilité d'esprit qui le caractérise. Les artistes joueront toujours un rôle important pour mettre en lumière les différences entre l'humain et la machine. 


\section{Références}

BOUCHER, Brian, « This is the Project of a More Just World : Trevor Paglen on Making Art That Show Alternative Realities » dans Arnet, 11 juin 2018.

En ligne, https://news.artnet.com/art-world/trevor-paglen-interview-1299836, page consultée le 05/02/2021.

CARPENTIER, Laurent. « L'autre terre de Grégory Chatonsky au Palais de Tokyo » dans Le Monde, 27 juin 2019. En ligne, https://www.lemonde.fr/ culture/article/2019/06/27/arts-l-autre-terre-de-gregory-chatonsky-au-palaisde-tokyo_5482045_3246.html, page consultée le 05/02/2021.

NORVELL, Patricia et Alexander Alberro. Recording Conceptual Art. Berkeley, Los Angeles et Londres, University of California Press, 2001. $177 \mathrm{p}$.

SMITHSON, Robert. The Collected Writings of Robert Smithson. Berkeley, Los Angeles et Londres, University of California Press, 1996. 424 p.

SOLNIT, Rebecca. Rivers of Shadow : Eadweard Muybridge and the Technological Wild West. New York, Penguin Books, 2004. 320 p. 